\title{
Fluoroless Radiofrequency Ablation of Typical Cavotricuspid Isthmus-dependent Atrial Flutter is a Safe and Practical Procedure
}

\author{
SANDEEP GAUTAM, MD ${ }^{1}$, RAHUL JAIN, MD, MPH ${ }^{2}$ and JOHN M. MILLER, MD ${ }^{2}$ \\ ${ }^{1}$ Division of Cardiovascular Medicine, University of Missouri, Columbia, MO \\ ${ }^{2}$ Division of Cardiology, Indiana University School of Medicine, Indianapolis, IN
}

\begin{abstract}
Electrophysiologic procedures, including radiofrequency ablation of cavotricuspid isthmus (CTI)-dependent atrial flutter, typically require varying amounts of fluoroscopy, which could be completely eliminated with electroanatomic mapping. We compared 20 consecutive patients who underwent fluoroless ablation for atrial flutter (Group A) with a prior series of 20 patients with standard flutter ablation (Group B). CARTO 3 mapping software was used to position diagnostic and ablation catheters in Group A; the ablation catheter was used to create a shell of the right atrium and coronary sinus (CS), and to guide CS catheter positioning. Atrial flutter was ablated successfully and bidirectional CTI block achieved without complication in all patients in both groups. Mean fluoroscopic duration and dose in Group B was $6.3 \pm 3.3$ min and $138 \pm 119 m$ Gy respectively. There was no difference between Group A and Group B in procedure duration (110.2 \pm 43.1 versus $114.9 \pm 29.2$ minutes, $p=0.69)$, ablation duration $(15.0 \pm 11.7$ versus $17.2 \pm 10.1 \mathrm{~min}, p=0.52$ ), time to ablation start ( $40.4 \pm 13.3$ versus $41.9 \pm 14.8 \mathrm{~min}$, $p=0.74)$, and time from CS catheter insertion to optimal placement (5.4 \pm 4.4 versus $6.3 \pm 5.0$ minutes, $p=0.74)$. There was also no significant difference in these parameters between the first and last 10 patients in Group A. Fluoroless ablation for right atrial CTI-dependent flutter is safe and practical, with obvious advantages of avoidance of radiation exposure to patients and staff. Further studies are warranted applying these principles to other arrhythmia substrates.
\end{abstract}

KEYWORDS. ablation, atrial flutter, fluoroscopy.
ISSN 2156-3977 (print) ISSN 2156-3993 (online)

(C) 2015 Innovations in Cardiac Rhythm Management

\section{Introduction}

Radiation exposure during ablation for cardiac arrhythmias has long been considered a "necessary evil." The deterministic and stochastic biological damage potential of radiation exposure during cardiac ablation procedures has been well documented. ${ }^{1}$ Fluoroscopic dose and exposure for

The authors report no conflicts of interest for the published content. John M. Miller reports fellowship funding, consulting and speaking honoraria from Biosense Webster, Inc.

Manuscript received November 22, 2015, final version accepted December 11, 2015.

Address correspondence to: Sandeep Gautam, MD, Assistant Professor of Clinical Medicine, Division of Cardiovascular Medicine, University of Missouri School of Medicine, One Hospital Drive, CE306, Columbia, MO 65212. E-mail: gautamsa@health.missouri.edu individual procedures can vary greatly depending upon the operator. Radiation exposure to the patient can vary from dose-area product $5-23 \mathrm{~Gy} \mathrm{~cm}^{2}$ for simple right-sided supraventricular arrhythmias, to $11-46 \mathrm{~Gy} \mathrm{~cm}^{2}$ for complex arrhythmias. ${ }^{2,3}$ In a recent survey of cardiac ablation and device placement procedures conducted by the European Heart Rhythm Association, only 50\% of the participating centers were found to consistently utilize low fluoroscopy frame rates, and the left anterior oblique fluoroscopy projection remained in common use, despite its known higher radiation exposure potential. ${ }^{4}$

Despite the advent of, and great improvement in, electroanatomic mapping (EAM) techniques over the last 20 years, fluoroscopy continues to be the primary imaging modality for intracardiac ablation procedures in the adult patient, although fluoroless (no fluoroscopy) ablation is being 
practiced in the pediatric population. ${ }^{5}$ The ALARA (as low as reasonably achievable) principle maintains that there is no measurable lower safe dose for radiation, and encourages "every reasonable effort to maintain exposures to ionizing radiation as far below the dose limits as practical." ${ }^{\prime 6}$ There have been some reports and case series of both simple and complex arrhythmias in adults being performed without fluoroscopy, mostly using a combination of three-dimensional EAM techniques, intracardiac echocardiogram (ICE), and remote magnetic navigation systems. ${ }^{7-9}$ However, use of fluoroless ablation remains extremely sporadic, most likely owing to concerns about procedural safety, efficacy, and efficiency.

The aim of the study was to evaluate the safety and efficacy of radiofrequency ablation (RFA) for typical cavotricuspid isthmus (CTI)-dependent atrial flutter performed in a fluoroless manner compared with the traditional method using fluoroscopy. We chose atrial flutter as it is a common supraventricular arrhythmia with a fairly standard ablation technique, and to limit the analysis to a single arrhythmia to ensure uniformity of data for comparison purposes.

\section{Materials and methods}

This is a retrospective study of patients who underwent RFA for typical CTI-dependent atrial flutter. We included the last 20 patients who underwent standard fluoroscopy-guided ablation for typical atrial flutter, and the first 20 patients who underwent fluoroless ablation for typical atrial flutter.

The primary aim of the study was to demonstrate procedural feasibility and compare procedural duration between standard fluoroscopy-guided ablation and fluoroless ablation.

In addition, the secondary aims included comparison of time to coronary sinus (CS) mapping catheter placement, ablation duration, time to start of ablation, procedural complications, and flutter recurrence over 12 months.

\section{Exclusion criteria}

Patients with cardiovascular implantable electronic devices were excluded from the study.

Patients were divided into the following two groups for descriptive purposes: Group A, 20 patients who underwent fluoroless RFA for typical atrial flutter; Group B, 20 patients underwent RFA for typical atrial flutter in the usual manner, utilizing fluoroscopy.

Total procedure duration was defined as duration from initial venous access to end of the procedure. Time to CS catheter placement was defined as the duration from advancing the CS catheter through the femoral venous sheath to adequate placement in the mid to distal CS. Time to ablation was described as the duration from initial femoral venous access to the first ablation lesion. Major bleeding was defined as requirement for blood transfusion, bleeding associated with a fall in hemoglobin $>2 \mathrm{~g} / \mathrm{dl}$, retroperitoneal bleeding, groin hematoma causing prolonged hospitalization or surgical intervention, or pericardial effusion requiring drainage. Minor bleeding was defined as groin hematoma not meeting the above criteria. Other perioperative complications such as stroke/transient ischemic attack and venous thromboembolic events were recorded in the database.

Patients were followed up in the outpatient clinic with electrocardiograms at 3,6 , and 12 months.

\section{Electrophysiology procedure details}

The patient was brought to the electrophysiology (EP) laboratory in a post-absorptive, non-sedated state. All procedures were performed under conscious sedation. Following adequate sedation, right femoral venous access was obtained twice following local anesthesia with $1 \%$ lidocaine, and 7-French and 8-French sheaths were advanced into this vein using a modified Seldinger technique. Both sheaths were flushed with heparinized saline.

Biosense Webster Carto 3 EAM software (Biosense Webster, Inc. Diamond Bar, CA, USA) was utilized in all cases. This system utilizes six location patches, three on the anterior chest and three posteriorly. For the purpose of the fluoroless procedures, the inferior-most back patches were positioned slightly more caudal than usual, in order to visualize the ablation catheter in the iliac veins and inferior vena cava. A 3.5-mm open irrigated-tip ThermoCool F curve ablation catheter (Biosense Webster, Inc. Diamond Bar, CA, USA) was used for mapping and ablation in all patients. The choice of a regular ThermoCool or surround flow (SF) ablation catheter was left to the discretion of the operator.

Electrophysiology procedure steps for Group A. The ablation catheter was advanced through the 8-French sheath, visualized using the EAM system, while maintaining a biplane left anterior oblique/right anterior oblique (LAO/ $\mathrm{RAO})$ projection on the EAM screen. The catheter was visualized through its entire course, and manipulated as required in the external iliac/inferior vena cava to negotiate any side branches. The catheter was advanced to the superior vena cava (SVC), and then withdrawn to the SVC/right atrial junction, confirmed by the presence of intra-atrial electrograms (EGMs) on only the proximal bipole. The catheter was deflected in an anterior and septal direction to localize the His bundle (position of His bundle was marked on CARTO), and then deflected further inferiorly and posteriorly, while staying septal, to localize the CS os. At this point, the catheter was gently rotated clockwise to enter the CS. The CARTO RAO view was switched to a posterior-anterior view to confirm CS location of the catheter, also confirmed by the intracardiac EGMs (Figure 1). Respiratory compensation training was performed at this location. The catheter was slowly withdrawn to the right atrium to create a detailed CS matrix, followed by creation of a right atrial matrix including the tricuspid annulus, inferior vena cava, and the CTI region. After completion of the right atrial "shell," a 20-pole Duodececapolar catheter (Biosense Webster, Inc. Diamond Bar, CA, USA) was advanced through the 7-French sheath to the right atrium, guided by the intracardiac EGMs. This catheter was visualized on EAM after entering the right atrial matrix, usually when the catheter tip was at or beyond SVC. From this point, the catheter was continuously visualized, and was 


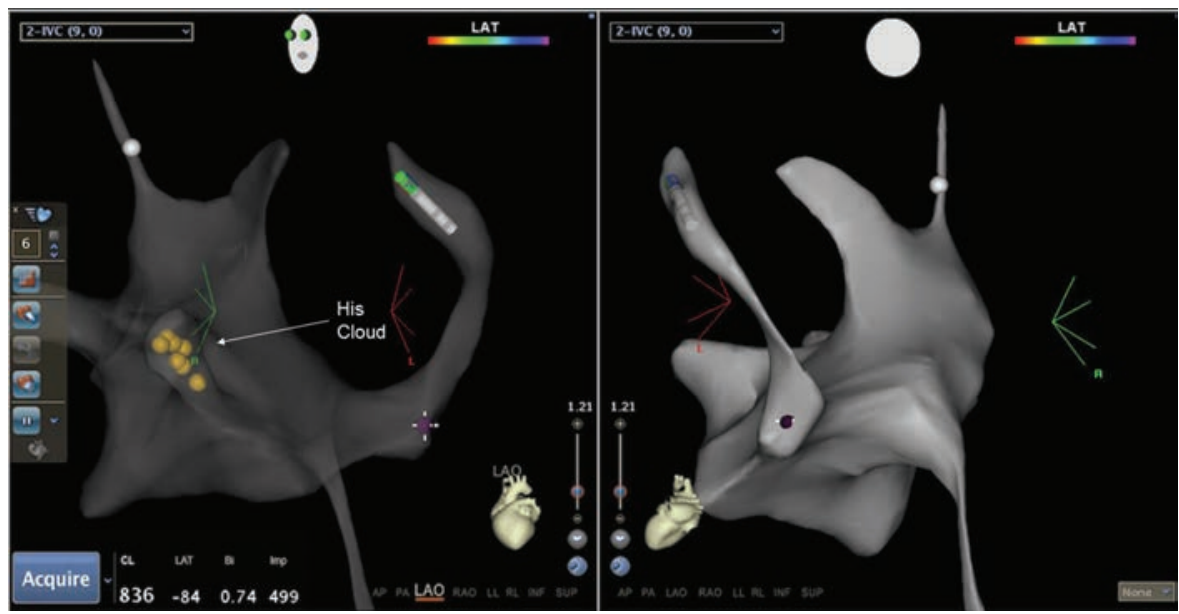

Figure 1: Fluoroless placement of ablation catheter in coronary sinus.

manipulated along the anterolateral right atrial wall to the mid-distal CS, using the previously created CS shell as a road map (Figure 2, Supplementary Video 1 and Video 2).

Electrophysiology procedure steps for Group B. A 20pole (2-8-2-(60)-2-8-2) Duodecapolar Biosense Webster (Biosense Webster, Inc. Diamond Bar, CA, USA), or Livewire (St Jude, St. Paul, MN, USA) catheter was advanced through the 7-French sheath under fluoroscopic guidance. This catheter was curved along the anterolateral wall of the right atrium, and advanced through the CS os into the distal CS, using a combination of RAO/LAO fluoroscopy. Catheter position was confirmed by fluoroscopy and intracardiac EGMs. The ablation catheter was advanced through the 8-French venous sheath, under fluoroscopic guidance, to the right atrium. A limited right atrial matrix was created, mainly for definition of the CTI region and visualization of the CS catheter on EAM.

RFA for CTI-dependent atrial flutter was performed in the usual manner, after confirmation of atrial flutter in patients who were in active arrhythmia during the procedure. RFA was started at the tricuspid annulus end of the mid-CTI region, gradually extending the ablation posteriorly to the inferior vena cava lip, until termination of atrial flutter (in patients with active flutter) and confirmation of bidirectional block across the isthmus by pacing from the proximal CS and the lateral right atrium using standard methods. The ablation catheter location was continuously monitored by EAM in both groups, and limited fluoroscopy in Group B (Figure 3). Intravenous adenosine, for confirmation of persistent bidirectional block, was used at the discretion of the operator. The procedure was considered

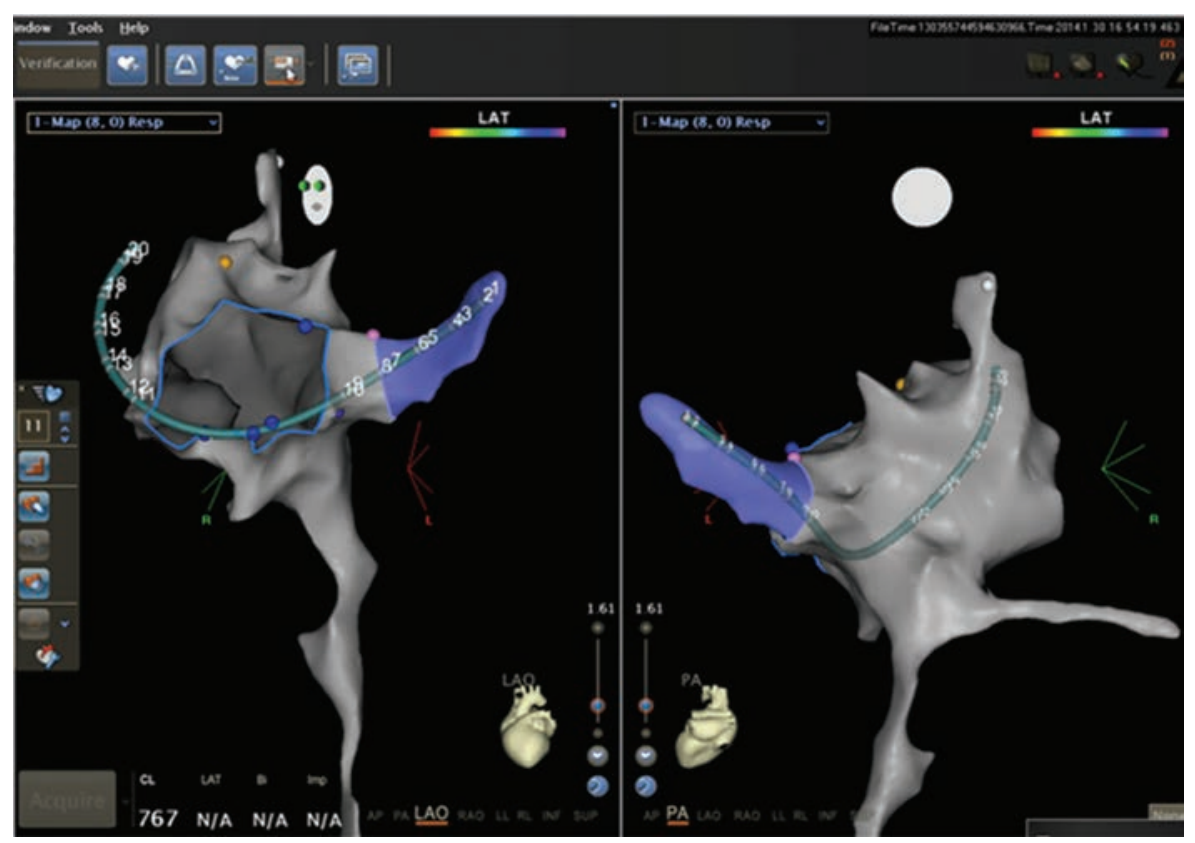

Figure 2: Fluoroless placement of Duodecapolar catheter in coronary sinus. 


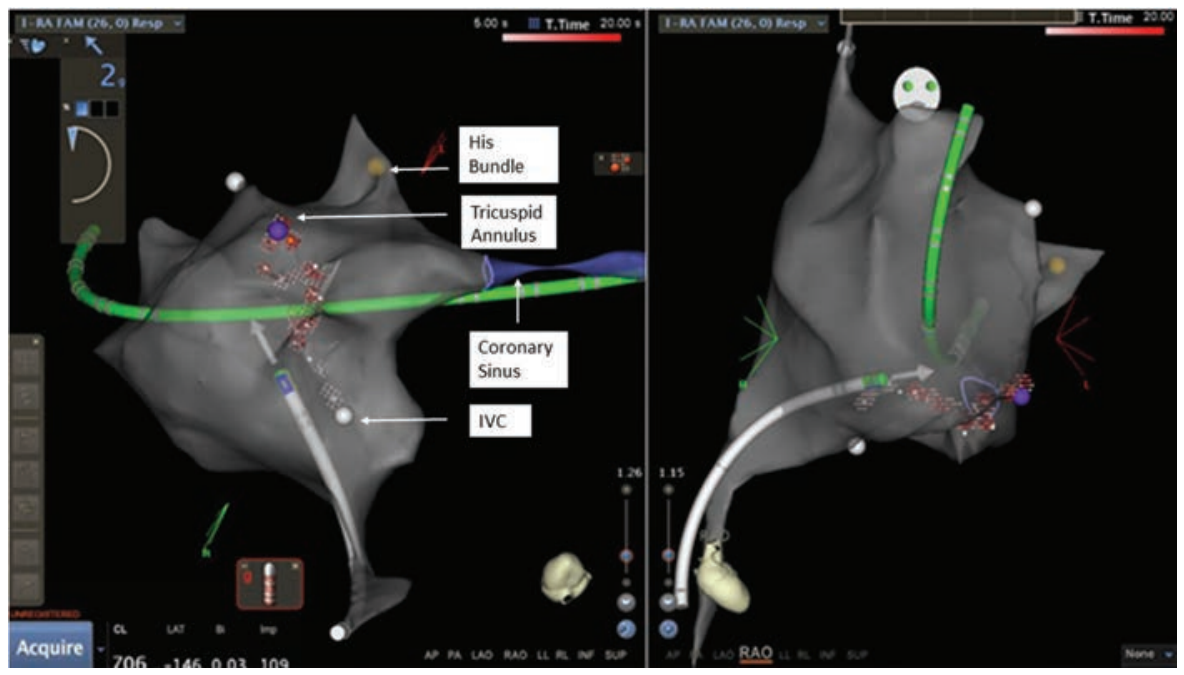

Figure 3: Fluoroless visualization of ablation and Duodecapolar catheters during ablation.

successful if bidirectional isthmus block was confirmed $30 \mathrm{~min}$ after the last ablation lesion, and absent or widely split EGMs > $100 \mathrm{~ms}$ were mapped over the ablation line. Both catheters were removed, followed by hemostasis in the usual manner.

One patient was excluded from Group A because of transient fluoroscopy for medication-induced hypotension.

\section{Statistical analysis}

Continuous variables are described as mean \pm standard deviation (SD) if normally distributed, or median and percentiles if not normally distributed. The data were compared between the two groups using the Student $\mathrm{t}$-test if normally distributed, or the Wilcoxon-Mann-Whitney rank sum test if not normal. Nominal and categorical variables were compared using the chi square or Fisher exact test. A value of $\mathrm{p}<0.05$ was considered significant. Stata 13 statistical software (StataCorp, College Station, TX) was used for statistical analysis.

\section{Results}

The two groups were well matched for baseline demographics, including age, sex, coronary artery disease (CAD), prior cardiac surgery, use of anticoagulants, history of atrial fibrillation, use of antiarrhythmic medications, and atrial flutter on presentation to the EP laboratory (Table 1). Novel oral anticoagulants (NOACs) were used more often in Group A. A long vascular sheath was required in 11 (55\%) patients in Group B to facilitate ablation, compared with none in Group A. A Livewire (St Jude, St. Paul, MN, USA) 20-pole catheter was used in $11(55 \%)$ patients in Group B. Bidirectional CTI isolation was performed successfully in all patients, without any procedural complications. Atrial flutter recurred in one patient in each group over the 12-month follow-up period.

\section{Procedural parameters in two groups}

Group A. No fluoroscopy was used in this group. Total procedure duration was $110.2 \pm 43.1 \mathrm{~min}$. Time to ablation start was $40.4 \pm 13.3 \mathrm{~min}$ (median 36.7 minutes), ablation duration $15.0 \pm 11.7 \mathrm{~min}$, and time to CS catheter placement $5.4 \pm 4.4 \mathrm{~min}$ (median $4.0 \mathrm{~min}$ ).

Group B. Mean fluoroscopic duration in this group was $6.3 \pm 3.3 \mathrm{~min}$, with an average fluoroscopic dose of 138.1 $\pm 119.3 \mathrm{mGy}$. Total procedure duration was $115.0 \pm 29.1 \mathrm{~min}$,

Table 1: Demographic comparison between Groups A and B

\begin{tabular}{lccc}
\hline & Group A & Group B & p-Value \\
\hline Age (years) & $66 \pm 13$ & $65 \pm 11$ & 0.64 \\
Weight (kg) & $96 \pm 24$ & $97 \pm 25$ & 0.44 \\
Male gender & $15(75 \%)$ & $15(75 \%)$ & 1 \\
CAD & $10(50 \%)$ & $10(50 \%)$ & 1 \\
CABG & $9(45 \%)$ & $8(40 \%)$ & 0.74 \\
Other cardiac surgery & $4(20 \%)$ & $4(20 \%)$ & 1 \\
Warfarin & $6(30 \%)$ & $11(55 \%)$ & 0.11 \\
NOAC & $10(50 \%)$ & $4(20 \%)$ & 0.047 \\
Atrial fibrillation & $7(35 \%)$ & $6(30 \%)$ & 0.73 \\
AAD medications & $5(25 \%)$ & $4(20 \%)$ & 0.7 \\
Flutter at Presentation & $12(60 \%)$ & $13(65 \%)$ & \\
\hline
\end{tabular}

AAD: antiarrhythmic drugs; CABG: coronary artery bypass graft; CAD: coronary artery disease; NOAC: novel oral anticoagulants. 
Table 2: Comparison of procedural parameters between Group A (fluoroless ablation) and Group B (regular ablation)

\begin{tabular}{lccc}
\hline & Group A $(\mathbf{n}=\mathbf{2 0})$ Mean \pm SD & Group B $(\mathbf{n}=\mathbf{2 0})$ Mean \pm SD & p-Value \\
\hline Procedure duration (minutes) & $110.2 \pm 43.1$ & $114.9 \pm 29.2$ & 0.69 \\
Ablation duration (min) & $15.0 \pm 11.7$ & $17.2 \pm 10.1$ & 0.519 \\
No of radiofrequency lesions & $30.8 \pm 23.1$ & $29.3 \pm 18.6$ & 0.822 \\
Time to CS placement (min) & $5.4 \pm 4.4$ & $6.3 \pm 5.0(\mathrm{n}=19)$ & 0.548 \\
Time to ablation start (min) & $40.4 \pm 13.3$ & $41.9 \pm 14.8$ & 0.739 \\
Fluoroscopy duration (min) & N/A & $6.3 \pm 3.3$ & $138.1 \pm 119.3$ \\
Fluoroscopy dose (mGy) & N/A &
\end{tabular}

of which time to ablation start was $41.9 \pm 14.8 \mathrm{~min}$, and ablation duration was $17.2 \pm 10.1 \mathrm{~min}$. Time to CS catheter placement was $6.3 \pm 5.0 \mathrm{~min}$ (median $4.5 \mathrm{~min}$ ). Time to CS catheter placement was not recorded in one patient in this group.

There was no statistically significant difference between any of the procedural parameters between the two groups (Table 2).

One patient in Group A required remapping of the right atrial matrix due to loss of Duodecapolar catheter visualization following patient movement.

We also compared the procedural parameters between the first 10 (Group A1) and later 10 (Group A2) patients who underwent fluoroless ablation, to study any effect of the "learning curve" on procedural efficiency (Table 3). As expected, procedural duration was shorter in Group A2, but this was not statistically significant. Time to CS catheter placement, time to ablation start, and ablation duration were similar between these groups.

\section{Discussion}

EAM has been available to facilitate electrophysiologic studies and ablation for the last 20 years. Over this time, the imaging resolution for EAM software has improved to $<1 \mathrm{~mm}$, similar to standard fluoroscopy. Almost all
EAM imaging software modules offer continuous cardiac structural mapping, which can be further refined with point-by-point mapping. With the accuracy offered by this modality, it should be a natural progression towards minimizing/completely eliminating fluoroscopy from most cardiac ablation procedures. However, although the use of fluoroless ablation of supraventricular arrhythmias is being aggressively pursued in the pediatric population, $5,10-13$ fluoroless ablation in the adult patient has been described in only a few studies from Europe, and with limited data from the United States.

Kerst et al. ${ }^{14}$ presented an observational series of 30 patients, including 12 children, 29 of whom underwent successful fluoroless ablation for right and left atrial arrhythmias using a contact force-sensing catheter, and ICE imaging for CS cannulation. Razminia et al. ${ }^{15}$ performed a retrospective analysis comparing 60 patients with a variety of arrhythmias who underwent ablation without fluoroscopy with a matched group of patient who had previously undergone standard ablation utilizing fluoroscopy. Direct comparison of procedural parameters was difficult in this study due to multiple arrhythmias in both groups. ICE imaging was used in all patients with CTI-dependent flutters to directly visualize the lesion line. The CS catheter was also placed using ICE guidance. Importantly, there were no complications related to lack of fluoroscopy. Reddy et al. ${ }^{8}$ and

Table 3: Comparison of demographics and procedural parameters between first 10 (Group A1) and second 10 (Group A2) patients in Group A

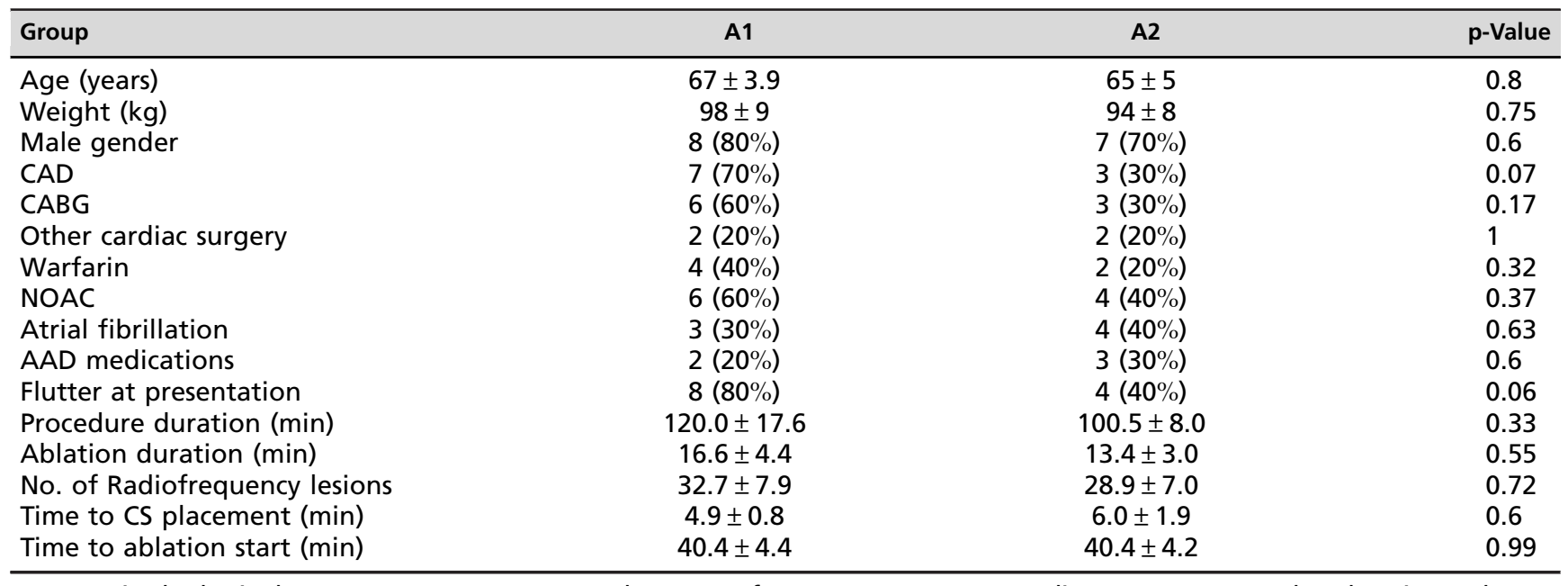

AAD: antiarrhythmic drugs; CABG: coronary artery bypass graft; CAD: coronary artery disease; NOAC: novel oral anticoagulants. 
Ferguson et al. ${ }^{16}$ have published case series of catheter ablation of atrial fibrillation without fluoroscopy, using a combination of ICE and three-dimensional EAM software. These studies demonstrated procedural safety. However, there was no comparative standard ablation group in these studies. Bulava et al. ${ }^{17}$ recently published a randomized trial of atrial fibrillation ablation (pulmonary vein isolation) with and without fluoroscopy in matched populations. They demonstrated equivalent procedural duration and success in both groups, without any serious complications. In this study, ICE imaging was used to enable CS cannulation with the diagnostic catheter, but additional atrial matrix creation was required for CS cannulation in $>50 \%$ patients.

Multiple studies have confirmed the procedural efficacy and safety of EAM in atrial flutter ablation, and demonstrated significant decrease in procedural radiation exposure. ${ }^{18}$ In particular, Hindricks et al. ${ }^{19}$ showed a $50 \%$ decrease in fluoroscopic time with EAM use in a prospective study of 210 atrial flutter ablations randomized to conventional or EAM-guided ablation. Schoene et al., ${ }^{20}$ in a randomized trial of atrial flutter ablation with or without a sensor-based non-fluoroscopic catheter tracking system (NFCT) (Mediguide ${ }^{\mathrm{TM}}$, St Jude Medical, Inc., St Paul, MN), showed a significant reduction in radiation dosage in the NFCT group without effect on procedure duration. This non-fluoroscopic technique requires acquisition of two cine loops, and is therefore not completely fluoroless. To our knowledge, to date, there is no published study on the effect of completely fluoroless atrial flutter ablation on procedural parameters and safety.

The unique points of our study are discussed below.

We believe that this is the first study to directly compare the procedural parameters for atrial flutter ablation with and without fluoroscopy, including procedure duration and particularly time for CS catheter placement. In contrast to previous studies, we did not use ICE to facilitate catheter manipulation and placement. The addition of ICE to atrial flutter ablation would significantly increase the procedural cost, with a small potential of complications related to the additional venous access for the ICE catheter.

Our study has demonstrated that the duration and efficacy of atrial flutter ablation procedure is not affected by lack of fluoroscopy. In particular, we have shown that the diagnostic and ablation catheters can be placed, and ablation completed, with equal efficiency and safety compared to standard fluoroscopy/EAM-guided atrial flutter ablation.

We have also shown that fluoroless ablation can be performed using EAM guidance, irrespective of the EAM software. To date, most studies have been performed with the St. Jude EnSite ${ }^{\circledR}$ (St Paul, MN) imaging software.

In addition to atrial flutter, we have successfully performed fluoroless ablations for right atrial tachycardias, AV node re-entrant tachycardia, right-sided accessory pathways, right/left ventricular tachycardias, and left atrial tachycardias via persistent foramen ovale. We believe that all of these procedures can be safely performed without fluoroscopy in any standard EP laboratory. We caution that fluoroscopy should be readily available in case of difficult vascular access or suspected procedural complications such as pericardial effusion

Our study did not include cost analysis for the use of EAM software. However, we do not consider this a deciding factor for use of fluoroless ablations, as most electrophysiologists routinely utilize EAM software to facilitate standard atrial flutter ablation. Although ablation can be performed without EAM, solely guided by fluoroscopy, these procedures typically result in higher fluoroscopic exposure, which is opposed to the ALARA recommendation.

\section{Study limitations}

This is a retrospective study, with the usual limitations regarding conclusions. Although the number of patients in our study is limited, we have intended this as a proofof-concept study, to demonstrate procedural feasibility and safety.

All procedures were performed by experienced operators, which could potentially affect the procedural duration and results. However, the learning curve for fluoroless flutter ablation was quite "steep," as evidenced by the lack of significant difference in procedural parameters between the first and second group of 10 patients who underwent fluoroless ablation.

Additional imaging modalities such as venous Doppler ultrasound could be needed/ utilized to enable easier vascular access, although not required in our study. As per the exclusion criteria, our findings are not applicable to patients with cardiac implantable electronic devices.

\section{Conclusions}

Most electrophysiologists continue to use fluoroscopy for ablation for CTI-dependent atrial flutter mainly due to concerns about procedural duration and efficacy. This retrospective study of RFA for typical atrial flutter demonstrates that the procedure can be safely and efficiently performed without the use of fluoroscopy. This finding has important implications for reduction of radiation exposure to patients as well as operators and staff during ablations. Further studies, such as the ongoing NO-PARTY trial, ${ }^{21}$ should be carried out about the cost effectiveness of this approach, and its utility in ablation of other cardiac arrhythmias.

\section{References}

1. Limbacher M, Douglas PS, Germano G, et al. ACC expert consensus document. Radiation safety in the practice of cardiology. American College of Cardiology. J Am Coll Cardiol. 1998;31(4):892-915.

2. Mettler Jr FA, Huda W, Yoshizumi TT, Mahesh M. Effective doses in radiology and diagnostic nuclear medicine: A catalog 1. Radiology. 2008;248(1):254-263.

3. Nof $E$, Lane C, Cazalas M, et al. Reducing radiation exposure in the electrophysiology laboratory: It is more than just fluoroscopy times! Pacing Clin Electrophysiol. 2015;38(1):136-145. 
4. Estner HL, Grazia Bongiorni M, Chen J, et al. Use of fluoroscopy in clinical electrophysiology in E: Results of the European Heart Rhythm Association survey. Europace. 2015;17(7):1149-1152.

5. Ebrille E, Caponi D, Siboldi A, et al. Single center experience of fluoroless AVNRT ablation guided by electroanatomic reconstruction in children and adolescents. Eur Heart J. 2013;34(Suppl. 1):P4974.

6. Huda W. Radiation risks: What is to be done? $A J R$ Am J Roentgenol. 2015;204(1):124-127.

7. Razminia M, Manankil MF, D'Silva O, et al. Non-fluoroscopic catheter ablation of cardiac arrhythmias in adults using electroanatomic mapping and intracardiac echocardiography. Circulation. 2011;124(21 Suppl.):A14518.

8. Reddy VY, Morales G, Ahmed H, et al. Catheter ablation of atrial fibrillation without the use of fluoroscopy. Heart Rhythm. 2010;7(11):1644-1653.

9. Scaglione M, Ebrille E, Francesca Di Clemente M, Gaita F. Catheter ablation of atrial fibrillation without radiation exposure using A 3D mapping system. J Atr Fibrillation. 2015;7(5):1-7.

10. Pass RH, Gates GG, Gellis LA, Nappo L, Ceresnak SR. Reducing patient radiation exposure during paediatric SVT ablations: Use of CARTO ${ }^{\circledR} 3$ in concert with "ALARA" principles profoundly lowers total dose. Cardiol Young. 2014;25(5):1-6.

11. Scaglione M, Ebrille E, Caponi D, et al. Single center experience of fluoroless AVNRT ablation guided by electroanatomic reconstruction in children and adolescents. Pacing Clin Electrophysiol. 2013;36(12):1460-1467.

12. Tuzcu V. Significant reduction of fluoroscopy in pediatric catheter ablation procedures: Long-term experience from a single center. Pacing Clin Electrophysiol. 2012;35(9):1067-1073.

13. Miyake CY, Mah DY, Atallah J, et al. Nonfluoroscopic imaging systems reduce radiation exposure in children undergoing ablation of supraventricular tachycardia. Heart Rhythm. 2011;8(4):519-525.

14. Kerst G, Weig H, Weretka S, et al. Contact force-controlled zero-fluoroscopy catheter ablation of right-sided and left atrial arrhythmia substrates. Heart Rhythm. 2012;9(5):709-714.

15. Razminia M, Manankil MF, Eryazici PL, et al. Nonfluoroscopic catheter ablation of cardiac arrhythmias in adults: Feasibility, safety, and efficacy. J Cardiovasc Electrophysiol. 2012;23(10):1078-1086.

16. Ferguson JD, Helms A, Mangrum JM, et al. Catheter ablation of atrial fibrillation without fluoroscopy using intracardiac echocardiography and electroanatomic mapping. Circ Arrhythm Electrophysiol. 2009;2(6):611-619.

17. Bulava A, Hanis J, Eisenberger M. Catheter ablation of atrial fibrillation using Zero-Fluoroscopy technique: A randomized trial. Pacing Clin Electrophysiol. 2015;38(7):797-806.

18. Casella M, Russo E, Pizzamiglio F, et al. The growing culture of A minimally fluoroscopic approach in electrophysiology lab. J Atr Fibrillation. 2014;7(2):1-11.

19. Hindricks G, Willems S, Kautzner J, et al. Effect of electroanatomically guided versus conventional catheter ablation of typical atrial flutter on the fluoroscopy time and resource use: A prospective randomized multicenter study. J Cardiovasc Electrophysiol. 2009;20(7):734-740.

20. Schoene K, Rolf S, Schloma D, et al. Ablation of typical atrial flutter using a non-fluoroscopic catheter tracking system vs. conventional fluoroscopy-results from a prospective randomized study. Europace. 2015;17(7):1117-1121.

21. Casella M, Dello Russo A, Pelargonio G, et al. Rationale and design of the NO-PARTY trial: Near-zero fluoroscopic exposure during catheter ablation of supraventricular arrhythmias in young patients. Cardiol Young. 2012;22(5): 539-546. 